\title{
Magnetotransport measurements on freely suspended two-dimensional electron gases
}

\author{
R. H. Blick,,${ }^{1, *}$ F. G. Monzon, ${ }^{1}$ W. Wegscheider, ${ }^{2, \dagger}$ M. Bichler, ${ }^{2}$ F. Stern, ${ }^{3, \sharp}$ and M. L. Roukes ${ }^{1}$ \\ ${ }^{1}$ Condensed Matter Physics 114-36, California Institute of Technology, Pasadena, California 91125 \\ ${ }^{2}$ Walter-Schottky-Institut der Technischen Universität München, Am Coulombwall, 85748 Garching, Germany \\ ${ }^{3}$ IBM T.J. Watson Research Center, P.O. Box 218, Yorktown Heights, New York 10598
}

(Received 10 April 2000; revised manuscript received 25 July 2000)

\begin{abstract}
We present magnetotransport measurements on freely suspended two-dimensional electron gases from $\mathrm{Al}_{x} \mathrm{Ga}_{1-x} \mathrm{As} / \mathrm{GaAs}$ heterostructures. The technique to realize such devices relies on a specially molecular beam epitaxy grown GaAs/ $\mathrm{Al}_{x} \mathrm{Ga}_{1-x} \mathrm{As}$-heterostructure, including a sacrificial layer. We fabricated simple miniHall-bars as well as quantum cavities and quantum dot systems. We find well-pronounced Shubnikov-de Haas oscillations and observe commensurability resonances, allowing characterization of the electron gas in these 100-nm thin membranes.
\end{abstract}

Recently the realization of suspended, monocrystalline, GaAs nanostructures containing a three-dimensional electron gas has been demonstrated. ${ }^{1}$ Previous work has shown the possibility of using a GaAs high-electron mobility transistor to achieve sensitive piezoelectric detection of strain. ${ }^{2} \mathrm{We}$ are combining these techniques to enable new means of detecting motion in nanomechanical systems and to study interactions in coupled electron-phonon systems of reduced dimensionality for both the electrons and the phonons. Freestanding structures incorporating a high-mobility electron gas have interesting device applications: A high mobility 2DEG system provides a unique approach to implement wideband, extremely sensitive displacement detection. These systems also offer prospects for very sensitive bolometers and represent model systems for high sensitivity calorimetry. ${ }^{3}$ One very interesting potential application is in heat-capacity measurements on two-dimensional electron gases (2DEGs). The sensitivity of these types of thermal devices is enhanced by their small volume, which can be of the order of $\sim 3 \mu \mathrm{m}^{3}$, significantly smaller than the usual dimensions of $\sim 10^{8} \mu \mathrm{m}^{3}$. 1,3

In this work we discuss the processing technique of such devices and present results on magnetotransport measurements on suspended two- and zero-dimensional systems. The processing employs a specially designed molecular-beam epitaxy (MBE)-grown 2DEG heterostructure in which a sacrificial layer is included. The layer structure is shown in Fig. 1(a): The structural layer stack, from which the devices are formed, comprises seven individual layers having a total thickness of $100 \mathrm{~nm}$. The top and bottom are formed by thin GaAs cap layers preventing oxidation of the $\mathrm{Al}_{x} \mathrm{Ga}_{1-x} \mathrm{As}$ : $\mathrm{Si}$ donor layers that follow beneath. The central 15-nm-thick GaAs layer forms a quantum well sustaining a high mobility 2DEG located $37 \mathrm{~nm}$ below the top surface and surrounded by two $\mathrm{Al}_{x} \mathrm{Ga}_{1-x} \mathrm{As}$ spacer layers. Below the structural layer stack is a $400 \mathrm{~nm} \mathrm{Al}_{0.8} \mathrm{Ga}_{0.2} \mathrm{As}$ sacrificial layer.

The heterostructure was designed by modeling the conduction-band lineup numerically. These calculations employed a Poisson-Schrödinger solver program, written by Laux and Kumar, which neglects many-body effects. ${ }^{4}$ In contrast to common 2DEG heterostructure configurations, here an additional GaAs layer is included to avoid deleteri- ous carrier depletion effects from the lower surface once it becomes exposed, i.e., after the sacrificial etch. The calculations indicate the donor regions are particularly susceptible to illumination, and that parallel conduction might be possible. However, in our measurements no obvious contribution from such channels even after continuous illumination was evident as will be discussed below. Hence, we only have to consider a single 2DEG layer in the evaluation below.

To realize these suspended nanostructures with threedimensional relief we employ multiple steps of optical and electron beam lithography, followed by a combination of pattern transfer steps. The latter involve both anisotropic ionbeam (dry) and chemically selective (wet) etching techniques. The first step of the processing procedure is fabrication of standard $\mathrm{Au} / \mathrm{Ni} / \mathrm{Ge}$ Ohmic contacts to the GaAs. These require an annealing step after their deposition. The second step is definition of contacts by optical and electron beam lithography and patterning with Ni. These contacts serve as a metallic etch mask during the subsequent ionbeam etch step. The Ni etch mask is selectively removed by another brief wet etch step employing $\mathrm{FeCl}_{3}$ after the dry etch. The device geometry is machined by anisotropic, chemically assisted ion-beam etching (CAIBE) with chlorine gas. The final step then is the selective chemical etch to remove the sacrificial layer. Depending on the stoichiometry of the structural layer, either a $2 \%$ solution of HF or concentrated $\mathrm{HCl}$ was employed. ${ }^{1,5}$

A typical freely suspended 2DEG sample is shown in Fig. 1(b): In this case the device has a shape similar to a conventional Hall bar with dimensions of $1 \times 2 \mu \mathrm{m}^{2}$ (width $\times$ length). In Fig. 2(a) a scanning electron microscope (SEM) micrograph of the same device as in Fig. 1(b) is shown, but taken at a steeper angle; the sacrificial layer is clearly seen to be completely removed from beneath the structure. In comparison to the usual Hall bars with a wide $2 \mathrm{DEG}$ region and small contact areas we had to choose a simplified design, since the underetching effectively limits the lateral extension of the system. For larger membranes the mechanical supports are commonly undercut as well, leading to a collapse of the whole structure. Although the contact areas are fairly large as compared to the device size, the mini-Hall-bar allows us to monitor the longitudinal voltage drop properly. 
(a)
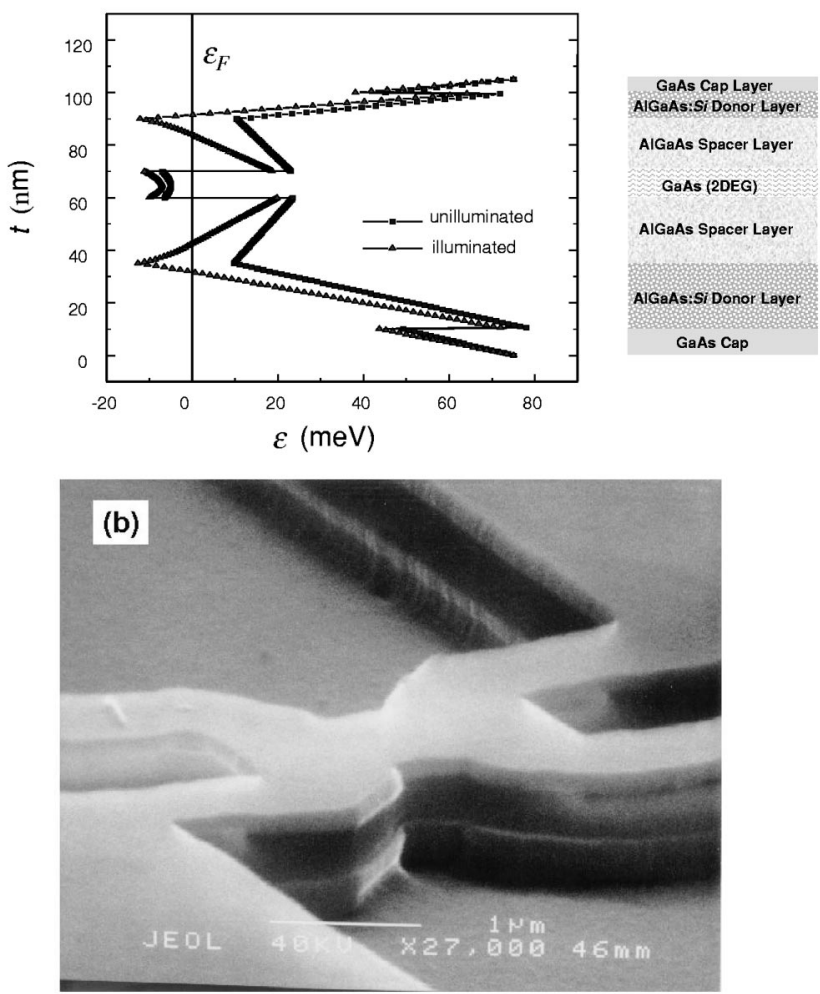

FIG. 1. (a) Calculation of the band structure of the freely suspended two-dimensional electron gas. As indicated, the filled squares mark the nonilluminated trace and the triangles the illuminated one. The Fermi level is pinned at midgap for top and bottom surfaces. The temperature assumed in the calculations is $T=10 \mathrm{~K}$. The top and bottom of the heterostructure are formed by GaAs cap layers, two $\mathrm{Al}_{x} \mathrm{Ga}_{1-x} \mathrm{As}: \mathrm{Si}$ donor layers and two $\mathrm{Al}_{x} \mathrm{Ga}_{1-x} \mathrm{As}$ spacer layers around the two-dimensional electron gas. (b) Scanning electron beam micrograph of the suspended mini-Hall-bar used in the measurements. The dimensions of this structure are length $\times$ width $=2 \times 1 \mu \mathrm{m}^{2}$, while the total thickness of the whole structure is $100 \mathrm{~nm}$.

Our quantum devices, also exemplified in Fig. 2(b), represent the first truly suspended quantum dots. The single dot has a final diameter of $800 \mathrm{~nm}$, while the diameter of the two coupled dots is of the order of $400 \mathrm{~nm}$. As discussed below, the actual electronic diameter of these devices is reduced by edge depletion. ${ }^{6}$ In the present case, coupling between the dots is mediated by the constriction regions connecting them. Careful design of the geometry of these regions allows us to control the carrier depletion and hence the degree of coupling to the leads. Fabrication of additional gate contacts in these regions, not attempted in these preliminary investigations, would permit definition of variable tunneling barriers.

At first, low-temperature magnetotransport measurements were carried out to characterize the electron gas in the miniHall-bar and the quantum devices. Figure 2(c) displays Shubnikov-de Haas ( $\mathrm{SdH}$ ) oscillations in the longitudinal resistance for one of the suspended Hall bar samples after illumination with a red light-emitting diode for $60 \mathrm{sec}$. Prior to illumination the resistance commonly was a factor of 5 larger, as seen in the values obtained for the cavities $A$ and $B$ in Fig. 3. The reason for this deviation might be also found
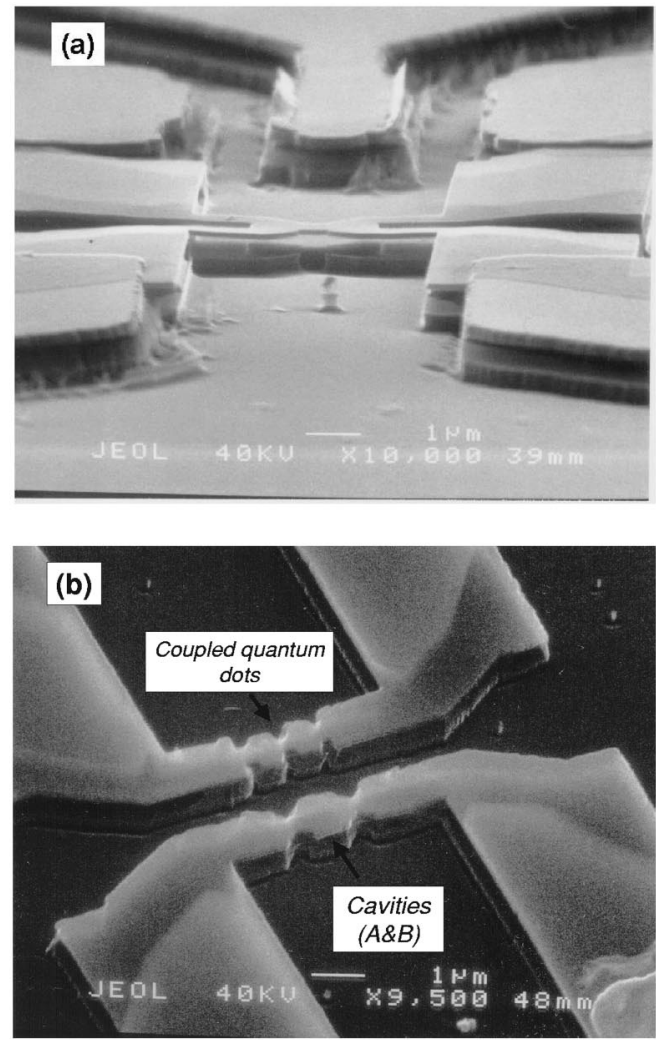

(c)

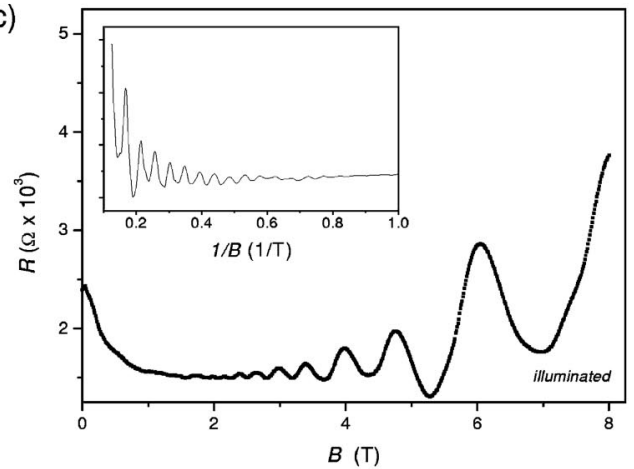

FIG. 2. (a) Side view of the device in Fig. 1(b), demonstrating the clear undercut; for all samples the same wafer material was used. (b) Aerial view of the suspended single and double quantum dots. The lithographical diameter of the single cavity is $800 \mathrm{~nm}$, while the double dots are of the order of $400 \mathrm{~nm}$. We fabricated several of these samples; data shown in Figs. 3 and 4 are obtained from two different cavities $A$ and $B$, while the data from the coupled dot is presented in Fig. 5. (c) Shown is the longitudinal magnetoresistance $R(B)$ of one of the suspended mini-Hall-bars at $T=4.2 \mathrm{~K}$ after illumination as indicated (the second sample's data is not shown). The inset gives a $(1 / B)$ plot demonstrating the periodicity of the oscillations after illumination (the electron density for samples used is obtained as $n_{s} \cong 5.5 \times 10^{15} \mathrm{~m}^{-2}$ ).

in a varying surface tension of the different suspended 2DEGs. The electron density is finally obtained from the $\mathrm{SdH}$ oscillations shown in Fig. 2(c) through the standard relation $\Delta(1 / B)=g_{s} e /\left(h n_{s}\right)$, where $g_{s}$ is the spin degeneracy factor (here $g_{s}=2$ ), which yields $n_{s} \sim 5.5 \times 10^{15} \mathrm{~m}^{-2}$. Note that the Landau levels are well developed even at fairly low fields. In the inset of Fig. 2(c) the (1/B) spectrum of the 

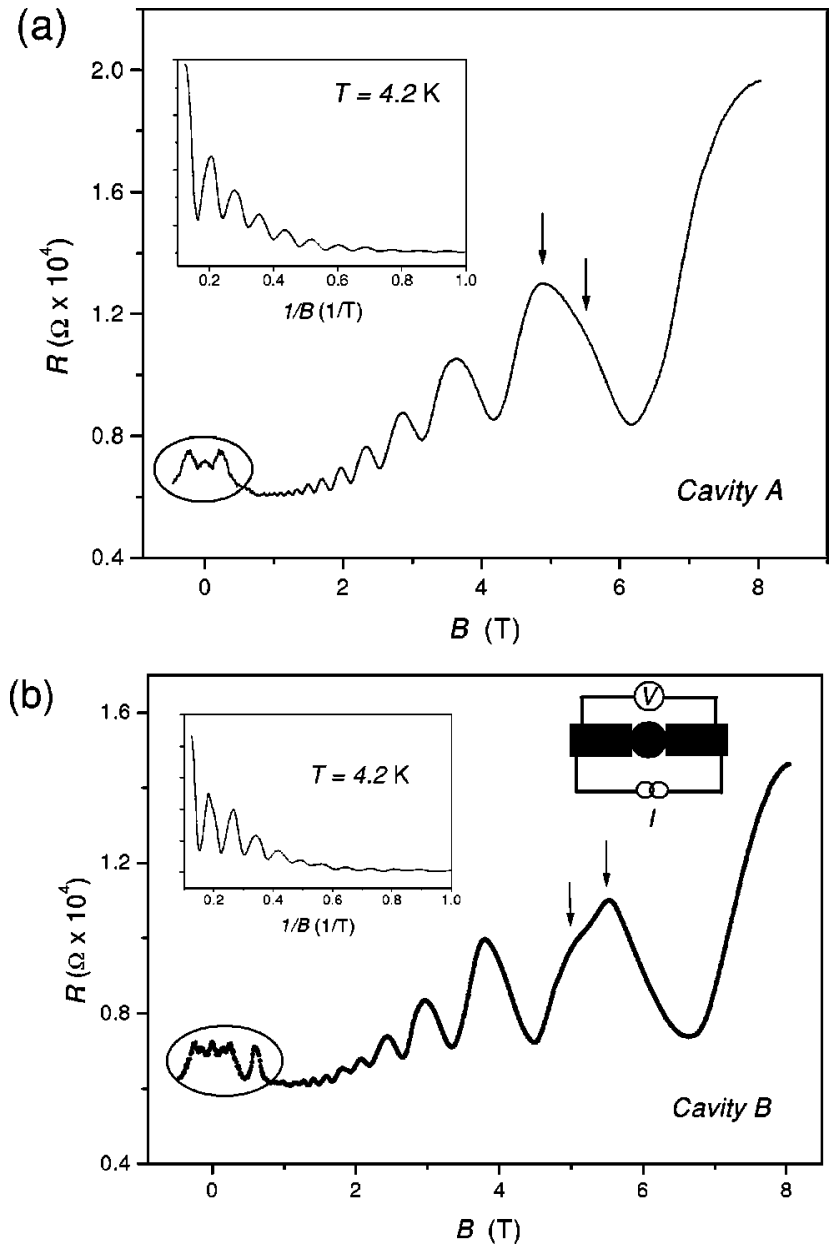

FIG. 3. (a) Magnetoresistance trace of the suspended "open", quantum dot (cavity $A$ ) prior to illumination (note the large resistance). The leads contain several transport channels, resulting in a strong coupling, i.e., $G>2 e^{2} / h$. In the high field region the classical Shubnikov-de Haas oscillations appear, while at low fields we find commensurability oscillations (circle). The two arrows mark the onset of the spin splitting. The inset gives a clear $1 / B$ periodicity of the oscillations; we obtain a density of $n_{s}^{c a v A}=5.65 \times 10^{15} \mathrm{~m}^{-2}$. (b) Corresponding Shubnikov-de Haas data of cavity $B$ (evaluation of the data in the inset gives $n_{s}^{\operatorname{cav} B}=6.38 \times 10^{15} \mathrm{~m}^{-2}$ ). For this cavity we find even better pronounced commensurability oscillations (circle). The inset depicts the experimental setup for the transport measurements. Also marked by arrows is the occurence of the first spin splitting.

oscillations for the illuminated case shows only a single period. Hence, a parallel conduction channel appears to be absent or can at least be neglected for the transport data, despite illumination and the model calculations. The most likely explanation for this is the fact that lattice strain effects are not included in the model calculations, but are expected to play a significant role for these suspended devices.

For the mini-Hall-bar the sheet resistance is of the order of $\rho=\rho_{x x} \sim 4 \mathrm{k} \Omega / \square$ without illumination, and $\sim 700 \Omega / \square$ $-800 \Omega / \square$ after illumination. The values are obtained by extrapolating the resistance values from $B=1.5 \mathrm{~T}$ to $B$ $\rightarrow 0 \mathrm{~T}$, in order to circumvent contributions by scattering around $B=0$ (seen in the strong negative magnetoresistance). As noted before, the length/width ratio of the Hall bars is $l / w=2 \mu \mathrm{m} / 1 \mu \mathrm{m}=2$. Combining this zero-field re- sistance of approximately $700 \Omega / \square$ with the measured carrier density, we obtain an electron mobility after illumination of $(1.4-1.6) \mathrm{m}^{2} / \mathrm{V} \mathrm{s}$. This is a factor of 27 below the value for the nonsuspended 2DEG. It is likely that this strong reduction of electron mobility results from the combined effects of damage imparted by ion bombardment during the dry etch step, and from inhomogeneous surface tension in the suspended structure [see Fig 2(a)]. This is currently under investigation. For future samples the etching process will be optimized in order to minimize damage to the device. ${ }^{7}$ At low magnetic fields negative magnetoresistance is manifested, indicating the prevalence of weak localization or interaction phenomena.

In Figs. 3(a) and 3(b) are shown the magnetoconductance traces of two different cavities with open leads as depicted in the SEM micrographs in Fig. 2(b). The devices constitute either quantum cavities or quantum dots, depending on the coupling to the leads, i.e., number of transmitting channels $N$ in the leads with a final conductance of $G=N 2 e^{2} / h$. We denote the two different structures measured as cavities $A$ and $B$, since the effective total resistance is far below the resistance quantum $h / e^{2}$. The measurements on the coupled dot device are discussed below (see Fig. 5). The density obtained from the $\mathrm{SdH}$ resonances at larger magnetic fields is $n_{s} \cong 5.5 \times 10^{15} \mathrm{~m}^{-2}$ (cavity $A$ ) and $n_{s} \cong 6.8 \times 10^{15} \mathrm{~m}^{-2}$ (cavity $B$ ). Here we do not take into account the spin degeneracy factor, since the spin splitting only emerges at $B \cong 4 \mathrm{~T}[\mathrm{cf}$. arrows in Figs. 3(a) and 3(b)]. The resulting mobilities are $\mu_{\text {cav } A, \text { cav } B}=0.4 \mathrm{~m}^{2} / \mathrm{V} \mathrm{s}$ and $0.3 \mathrm{~m}^{2} / \mathrm{V} \mathrm{s}$, strongly reduced by the barriers introduced (compared to the "free", 2DEG). As seen, the data at larger fields exhibit the common signature of a 2DEG, while at lower fields an intricate resonance structure is found.

In focusing on this fine structure around $B=0 \mathrm{~T}$, shown in Fig. 4, we are able to extract some of the properties of the electron gas in the suspended membrane: The low temperature measurement at $4.2 \mathrm{~K}$ yields two distinct features, namely the coarse modulation of the resistance and a fine structure with a modulation period of $\delta B_{\text {cavA }}=(26.1$ $\pm 1.0) \mathrm{mT}$. This fine structure vanishes rapidly upon increasing the temperature [see traces for $10 \mathrm{~K}$ in Figs. 4(a) and 4(b) ]. Under illumination the carrier density varies and the $B$ periodicity disappears, even at $4.2 \mathrm{~K}$ [Fig. 4(a)].

Evaluating the periodicity of the fine structure by treating it as Aharonov-Bohm (AB) oscillations, we obtain a radius of the first cavity of $r_{A B}^{c a v A}=\sqrt{\phi_{0} /\left(\pi \times \delta B_{\text {cavA }}\right)}$ $=\sqrt{4.14 \times 10^{-15} \mathrm{~T} \mathrm{~m}^{2} /(\pi \times 20 \mathrm{mT})} \approx 225 \mathrm{~nm}$ and with $\delta B_{\text {cav } B}=(26.3 \pm 0.5) \mathrm{mT}$ for the second one $r_{A B}^{\text {cav } A}$ $=220 \mathrm{~nm}$. With this value we obtain an "electronic" diameter of the cavity of $450 \mathrm{~nm}$ for the first cavity $(A)$ and 440 $\mathrm{nm}$ for the second cavity $(B)$. Compared to the lithographically defined size of $800 \mathrm{~nm}$, we thus find a depletion depth of $\sim 175 \mathrm{~nm}$ from the edges. The fact that AB-like oscillations are found without an opening in the center could possibly be explained by some local inhomogeneity within the dot, e.g., local strain in the 2DEG, which might result in an effective depletion of the electrons in the center of the cavity. This also agrees with the disappearance of $\mathrm{AB}$ oscillations after illumination (higher density). Apart from the pecularity of the existence of $\mathrm{AB}$ oscillations in such a sample it has to 

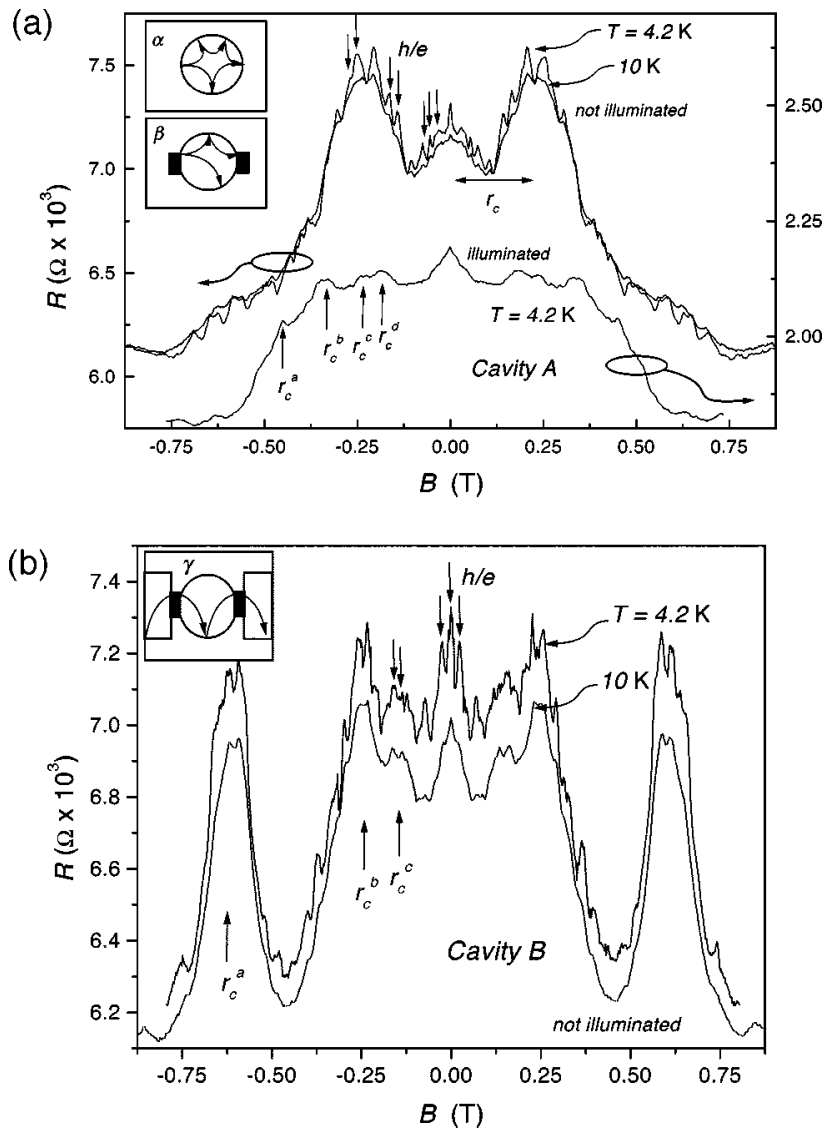

FIG. 4. (a) At the lowest temperatures $(4.2 \mathrm{~K}$ plot $)$ we find commensurability resonances and superimposed $B$-periodic Aharonov-Bohm-type oscillations from which we derive an "electronic" diameter of the cavity of $\sim 450 \mathrm{~nm}$ for cavity $A$. The oscillations clearly vanish at $10 \mathrm{~K}$ and under illumination. The geometric resonances are also modified under illumination. The commensurability resonances equally allow the determination of the cavity diameter: Calculating the cyclotron radii we find $r_{c}$ $=652 \mathrm{~nm}, r_{c}^{d}=680 \mathrm{~nm}, r_{c}^{c}=484 \mathrm{~nm}, r_{c}^{b}=357 \mathrm{~nm}, r_{c}^{a}=265 \mathrm{~nm}$ (see text for details). The insets give some of the possible orbits: The upper sketch $\alpha$ shows the ideal case, while in the lower one $\beta$ we allowed a finite contact diameter, demonstrating the occurence of the different cyclotron radii. (b) Similar commensurability oscillations and almost identical fine structure for cavity $B$. Calculating the radii of the cyclotron orbits yields $r_{c}^{c}=874 \mathrm{~nm}, r_{c}^{b}=539 \mathrm{~nm}$, $r_{c}^{a}=210 \mathrm{~nm}$. The inset $(\gamma)$ sketches a possible electron trajectory with maximum cyclotron radius.

be noted that dissipation phenomena in free-standing 2DEGs seem to be less pronounced than in comparable 2DEGs connected to the bulk GaAs crystal.

The electronic radius found from the AB-like oscillations is confirmed by the coarse modulation, which can be explained by geometric resonances found earlier in strongly modulated one-dimensional electron systems. 8,9 From this model we obtain the classical cyclotron radius $r_{c}=\hbar k_{F} / e B$ with $k_{F}=\sqrt{2 \pi n_{s}}$ being the Fermi wave vector. For the two quantum cavities measured (Fig. 3) we find a variety of different radii: For the first cavity $A$ [Fig. 4(a)] we obtained $r_{c}=652 \mathrm{~nm}, r_{c}^{d}=680 \mathrm{~nm}, r_{c}^{c}=484 \mathrm{~nm}, r_{c}^{b}=357 \mathrm{~nm}$, and $r_{c}^{a}=265 \mathrm{~nm}$ after illumination. Prior to illumination the only cyclotron orbit found is $r_{c}=r_{c}^{c}$. Similar commensurability oscillations and an almost identical fine structure are found

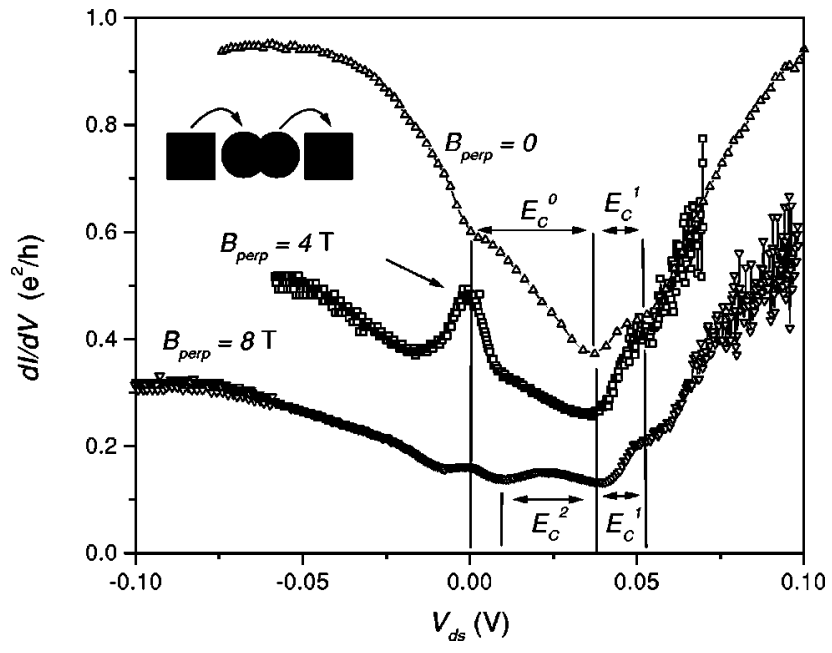

FIG. 5. Drain and/or source conductance of the coupled dot in the tunneling regime for different magnetic fields at $4.2 \mathrm{~K}$. Marked with $E_{C}^{0}, E_{C}^{1}$, and $E_{C}^{2}$ are the different possible charging states, changing upon increasing the field (see text for details). The inset indicates sequential electron tunneling through the coupled dot.

for cavity $B$ [Fig. 4(b)]: $r_{c}^{c}=874 \mathrm{~nm}, r_{c}^{b}=539 \mathrm{~nm}$, and $r_{c}^{a}$ $=210 \mathrm{~nm}$. The insets in Figs. 4(a) and 4(b) show some of the possible orbits: The upper sketch $\alpha$ shows the ideal case, while in the lower one $(\beta)$ we allowed a finite contact width, demonstrating the occurence of the different cyclotron radii. In the third case $\gamma$ the electron follows a trajectory with maximum cyclotron radius (with non ideal contacts), elucidating the large values of 600-800 $\mathrm{nm}$ measured. Considering the different radii and the corresponding orbits we obtain for the smallest radii $A, r_{c}^{a}=265 \mathrm{~nm}$, and for $B r_{c}^{a}$ $=210 \mathrm{~nm}$. Since we find similar radii for the commensurability oscillations, being caused by the coarse modulation, and under the assumption of $\mathrm{AB}$ oscillations, caused by the fine structure, we are confident that the depletion depth is correctly determined.

Finally, we want to present first transport data on suspended quantum dots as shown in Fig. 2(b), i.e., for the case when $G<e^{2} / h$. Already strong indications were found that the relaxation mechanisms of single electrons tunneling through coupled dots are influenced by discrete phonon modes. ${ }^{10,11}$ However, these quantum dots were still embedded in the bulk GaAs crystal and hence the phonon coupling is not well controlled. The conductance measurements on a quantum dot under bias voltage strongly decoupled from the leads are shown in Fig. 5. For these measurements we employed the coupled dot system shown in Fig. 2(b), since the smaller total size and the narrower contacts result in an increased resistance. As seen we find a transition into the tunneling regime for $B=0 \mathrm{~T}$ in the low drain and/or source bias $\left(V_{d s}< \pm 75 \mathrm{mV}\right)$ regime where the conductance $G$ $=d I / d V$ is found to be below $e^{2} / h$. The conductance drops below $e^{2} / h$ for bias voltages around +20 to $+50 \mathrm{mV}$. This drop is found to occur stepwise. Including the magnetic field dependence we are able to identify three different step widths, which are denoted $E_{C}^{0}, E_{C}^{1}$, and $E_{C}^{2}$. Interpreted as charging states of the quantum dots these steps correspond to energies of approximately 40, 10, and $30 \mathrm{meV}$, respectively. A crude estimation of the charging energy expected from the 
electronic dot radius of roughly $r_{e} \sim 200 \mathrm{~nm}$ (using the values found above for the depletion depth) we obtain $E_{C}$ $=e^{2} / 2 C_{\Sigma} \sim 1 \mathrm{meV}$. We assumed a dielectric disk with $C_{\Sigma}$ $=8 \epsilon_{0} \epsilon_{r} r_{e}$, where $C_{\Sigma}$ is the total capacitance of the dot, $\epsilon_{r}$ the dielectric constant of $\mathrm{Al}_{x} \mathrm{Ga}_{1-x} \mathrm{As}$ and $\epsilon_{0}=8.85$ $\times 10^{-12} \mathrm{~F} / \mathrm{m}$. Although this value is far below the stepsize found in Fig. 5, it is still sufficient to allow Coulomb blockade $(\mathrm{CB})$ at $4.2 \mathrm{~K}$. Moreover, it is possible that the electronic diameter of the coupled dots is smaller than we estimated here.

Unfortunately, it was not possible to verify charging of the quantum dot device by an external gate directly. Thus no clear CB signature can be identified for this device. Instead we applied a magnetic field perpendicular to the plane of the dot. Increasing the field, we observe a suppression and an additional modulation of the conductance around $V_{d s}=0$, as seen for $B=4 \mathrm{~T}$ and $8 \mathrm{~T}$. Discrete states in the quantum dot must have formed, indicated by the structure in the $I V$ characteristic of Fig. 5. This is evidenced by the modulation at finite magnetic field. Additionally, we find an enhanced background noise when keeping the dots under forward bias, which can be attributed to an effective mechanical vibration of the whole sample, due to the resulting Lorentz force in the magnetic field.

In summary, we have demonstrated a technique of how to fabricate freely suspended two-dimensional electron gases.
In transport measurements we observe the characteristic features of 2DEGs. Moreover, we find at low temperatures a strong negative magnetoresistance effect, indicating the lower mobilities of the suspended electron gas compared to the bulk values. In further measurements on suspended quantum dots and quantum cavities we found geometric resonances and a fine structure from which we estimate the lateral depletion length to be $175 \mathrm{~nm}$. Finally, we presented first measurements of transport spectroscopy on a suspended quantum dot structure revealing the existence of discrete states. Although the properties of the suspended electron gas are not yet optimized in comparison to conventional $\mathrm{Al}_{x} \mathrm{Ga}_{1-x} \mathrm{As} / \mathrm{GaAs}$ heterostructures, we clearly demonstrated functioning of these devices. Moreover, this technique offers a broad variety of possible applications, including highfrequency strain sensing in nanomechanical devices, studies on single electron-phonon interaction, measurements of electronic specific heat, and ultrasensitive bolometry.

We gratefully acknowledge funding for this work from DARPA MTO/MEMS under Grant No. DABT-63-98-10012. We thank A.N. Cleland for expert technical help and S. Ulloa for critically reading the manuscript. R.H.B. thanks the Max-Planck-Gesellschaft (Otto-Hahn stipend), the MaxPlanck-Institute for Solid State Physics, Stuttgart, and the Alexander-von-Humboldt Stiftung (Feodor-Lynen stipend) for support.
*Current address: Center for NanoScience and Sektion Physik, Ludwig-Maximilians-Universität München, Geschwister-SchollPlatz 1, 80539 Munich, Germany.

Email: robert.blick@physik.uni-muenchen.de

†New address: Institut für Angewandte und Experimentelle Physik, Universität Regensburg, 93040 Regensburg, Germany.

IIBM Research Staff Member Emeritus.

${ }^{1}$ T.S. Tighe, J.M. Worlock, and M.L. Roukes, Appl. Phys. Lett. 70, 2687 (1997).

${ }^{2}$ R.G. Beck, M.A. Eriksson, R.M. Westervelt, K.L. Campman, and A.C. Gossard, Appl. Phys. Lett. 68, 3763 (1996); 73, 1149 (1998)

${ }^{3}$ K. Schwab, E.A. Henriksen, J.M. Worlock, and M.L. Roukes, Nature (London) 404, 974 (2000).

${ }^{4}$ A. Kumar, S.E. Laux, and F. Stern, Phys. Rev. B 42, 5166 (1990).
${ }^{5}$ H. Blauvelt, N. Bar-Chaim, D. Fekete, S. Margalit, and A. Yariv, Appl. Phys. Lett. 40, 289 (1982).

${ }^{6}$ K.K. Choi, D.C. Tsui, and K. Alavi, Appl. Phys. Lett. 50, 110 (1987).

${ }^{7}$ H.X. Tang, F.G. Monzon, R.H. Blick, M.L. Roukes, M. Bichler, and W. Wegscheider (unpublished).

${ }^{8}$ T.J. Thornton, M.L. Roukes, A. Scherer, and B.P. Van de Gaag, Phys. Rev. Lett. 63, 2128 (1989).

${ }^{9}$ G. Müller, P. Streda, D. Weiss, K. von Klitzing, and G. Weimann, Phys. Rev. B 50, 8938 (1994).

${ }^{10}$ T. Fujisawa, T.H. Oosterkamp, W.G. van der Wiel, B.W. Broer, R. Aguado, S. Tarucha, and L.P. Kouwenhoven, Science 282, 932 (1998).

${ }^{11}$ T. Brandes and B. Kramer, Phys. Rev. Lett. 83, 3021 (1999). 